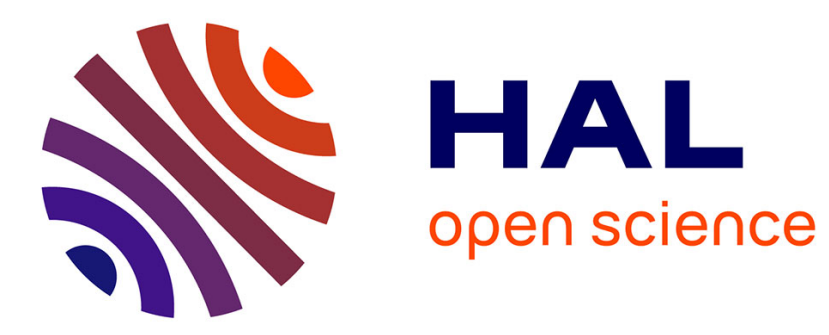

\title{
Automated Detection of Streaks in Dermoscopy Images
}

\author{
K. Delibasis, K. Kottari, I. Maglogiannis
}

\section{To cite this version:}

K. Delibasis, K. Kottari, I. Maglogiannis. Automated Detection of Streaks in Dermoscopy Images. 11th IFIP International Conference on Artificial Intelligence Applications and Innovations (AIAI 2015), Sep 2015, Bayonne, France. pp.45-60, 10.1007/978-3-319-23868-5_4 . hal-01385344

\section{HAL Id: hal-01385344 \\ https://hal.inria.fr/hal-01385344}

Submitted on 21 Oct 2016

HAL is a multi-disciplinary open access archive for the deposit and dissemination of scientific research documents, whether they are published or not. The documents may come from teaching and research institutions in France or abroad, or from public or private research centers.
L'archive ouverte pluridisciplinaire HAL, est destinée au dépôt et à la diffusion de documents scientifiques de niveau recherche, publiés ou non, émanant des établissements d'enseignement et de recherche français ou étrangers, des laboratoires publics ou privés. 


\title{
Automated Detection of Streaks in Dermoscopy Images
}

\author{
K. Delibasis ${ }^{1}$, K. Kottari ${ }^{1}$, I. Maglogiannis ${ }^{2}$ \\ ${ }^{1}$ Dept. of Computer Science and Biomedical Informatics, Univ. Of Thessaly, Greece \\ ${ }^{2}$ Department of Digital Systems, University of Piraeus, Greece \\ \{kdelibasis, kottarikonstantina\} @gmail.com \\ imaglo@unipi.gr
}

\begin{abstract}
In this paper we present a novel algorithm for the detection of dark linear structures, which appear in digital dermoscopy images of skin lesions and they are called as streaks in relevant literature. The proposed algorithm is capable of detecting such linear structures using local image curvature information obtained by the Hessian matrix. A linear structure is characterized as streak, based on its geometric characteristics. The streak detection algorithm is applied to a number of dermoscopy images containing malignant and non-malignant skin lesions. In this work we propose also a new class of streak based image features and we investigate their value in image classification according to malignancy.
\end{abstract}

Keywords: Computer vision, Skin lesions, Image Segmentation, Streaks detection, Dermoscopy images, Melanoma classification

\section{$1 \quad$ Introduction}

Skin cancer is considered among the most frequent types of cancer and one of the most malignant tumors. According to a recent study, its incidence has increased faster than that of almost all other cancers among young adults [1]. Several additional reports [2], [3], [4] have proven the importance of the early diagnosis in skin cancer related diseases and especially the melanoma cases. Thus Dermoscopy or Dermatoscopy, which is the examination of skin lesions or disorders through the use of the dermatoscope, a medical instrument capable of magnifying the examined area of the skin, has been widely used. This technique can reveal many color and structural features of the skin lesion, which would be impossible to observe with a naked eye. In addition advanced computer based systems can be used to capture a digital image produced by the dermatoscope, then analyze it by checking its morphological and color characteristics and extract measurable features. Such features could feed an expert system for providing an evaluation about the malignancy of the lesion [5], [6]. Methodologies and rules applied in the classic dermoscopy, can also be utilized in computer based dermoscopy. More specifically, in the conventional procedure, the 
following diagnostic methods are mainly used: (i) ABCD rule, (ii) Menzies rule, (iii) 7-point checklist and (iv) Pattern and Texture Analysis. For instance the ABCD rule investigates the asymmetry (A), border (B), color (C), and differential structures (D) such as Pigment Network and Streaks of the lesion and defines the basis for a diagnosis by a dermatologist. According to the published overviews [5], [6].

It is evident that the emphasis has been on assessment of lesion size, shape, color, and texture of lesions. Differential structures, especially streaks, which are distinct dark linear structures, seem to have been neglected by the computer vision community, although they are considered essential for manual lesion categorization. Streaks are brownish-black linear structures of variable thickness, not clearly combined with pigment network lines, mostly at the periphery of the lesion. Irregular streaks are very likely to represent melanoma, especially when the streaks are distributed unevenly. The detection of streaks in dermoscopy images has been recently reported in [7], [8].

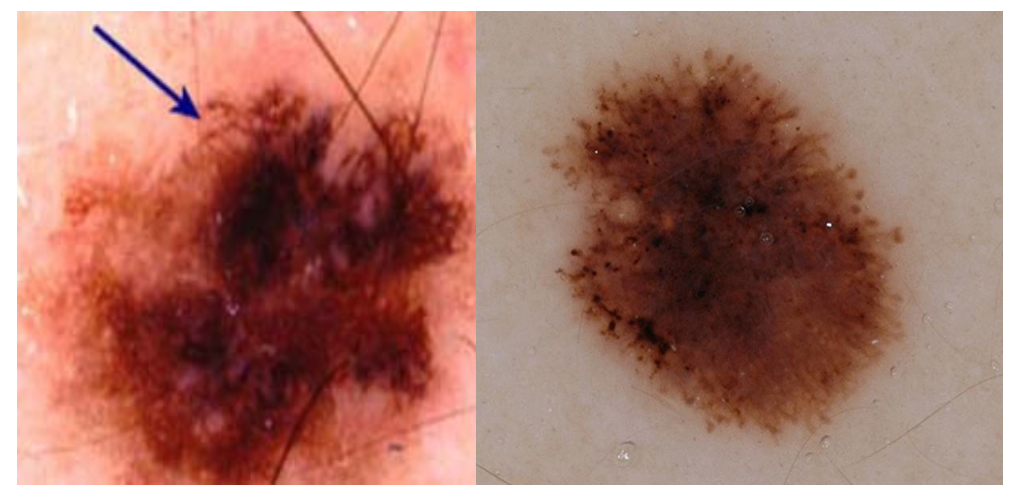

Fig. 1. Examples of images with streaks

In this work, we concentrate on the detection/segmentation of streaks utilizing advanced image processing techniques. Our work involves a simpler approach than the one described in [8]. Furthermore we attempt to evaluate the potential usefulness and diagnostic value of streaks for the differentiation of dermoscopy images into melanoma and non-malignant lesions. More specifically at a first stage we propose a novel algorithm for the segmentation of streaks in dermoscopy images, using the Hessian matrix to obtain local information about the image curvature. Dark linear structures are detected at different scales and their geometric characteristics are used to discriminate between not-streak structures. At a second stage we propose new features based on streaks existence, localization and distribution and investigate their value in computer based dermoscopy image analysis. The rest of the paper is structured as follows: In Section 2 we describe the technical details of proposed methodology for streaks segmentation and features extraction. Section 3 presents the initial experimental results and Section 4 concludes the paper. 


\section{Methodology}

The proposed algorithm involves a number of steps, which are illustrated in Fig. 2. Firstly, the dark linear structures are identified and segmented using hessian-base multiresolution approach and hysteresis thresholding. The segmented linear structures are labeled and their geometric characteristics (curvature, position and orientation) are then calculated. Those that satisfy the predefined criteria are labeled as streaks. A number of streak-based features are extracted and used for classification of each dermoscopy image according to malignancy. Each step of the proposed algorithm is presented in detail in the following subsections.

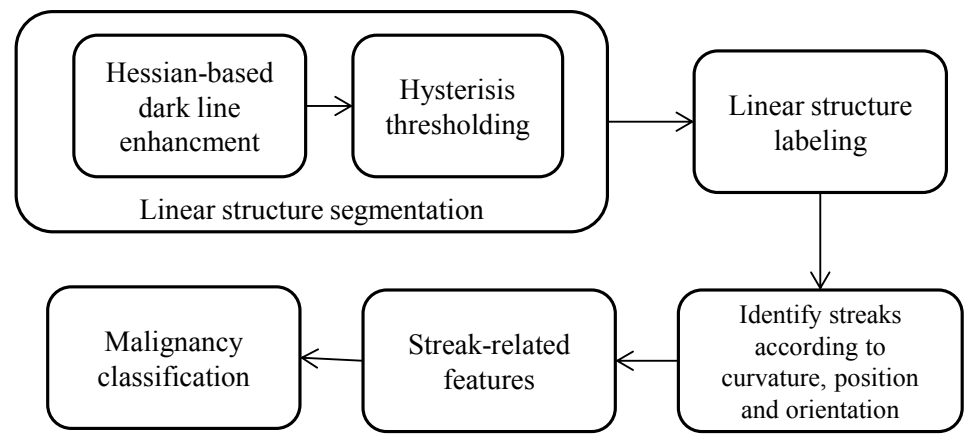

Fig. 2. Brief outline of the proposed algorithm

\subsection{Segmentation of linear structures in images}

In this subsection, we describe an algorithm for the segmentation of the dark linear structures in dermoscopy images. The Hessian matrix is used as a measure of linearity for each pixel $(x, y)$ of image $I$. The use of the Hessian matrix for image feature extraction has been reported frequently in the literature [9], [10]. In [11], [12], the eigenvalues of the Hessian matrix have been used to segment vessels. Let us define the Hessian matrix as following:

$$
\mathbf{H}(x, y ; \sigma)=\left(\begin{array}{ll}
I_{x x}(x, y) & I_{x y}(x, y) \\
I_{x y}(x, y) & I_{y y}(x, y)
\end{array}\right)
$$

, where the subscript denotes partial differentiation with respect to the corresponding image axis. The image partial derivatives in $\mathbf{H}$ are calculated using linear convolution (denoted by $*$ ) with the partial derivatives of a 2D Gaussian function, with variance $\sigma$ :

$$
\begin{gathered}
G(x, y ; \sigma)=\left(\frac{1}{\sqrt{2 \pi} \sigma}\right)^{2} e^{-\frac{x^{2}+y^{2}}{2 \sigma^{2}}} \\
I_{x x}(x, y ; \sigma)=\sigma^{y} I(x, y) * \frac{\partial^{2} G(x, y ; \sigma)}{\partial x^{2}}
\end{gathered}
$$


The $\sigma$ parameter is used to select image features at different scales, based on matrix $\mathbf{H}$, depending on the spatial resolution of the dermoscopy image. This is a wellreferenced technique [12], [13]. The selected values for $\sigma$ are discussed in the result section. The image partial derivatives, as described in (3) are normalized with respect to $\sigma$ by multiplying them with the term $\sigma^{\gamma}$, as described in [11], [14], so that the resulting values for different values of $\sigma$ are directly comparable. In this work, we set $\gamma=1$. The Hessian matrix has been frequently utilized to describe the local structure of an image. In [11] a number of types of image points are defined according to the eigenvalues of $\mathbf{H}$, in 2 and in 3 dimensions. In [15] the Hessian matrix is used to define the diffusivity function in order to enhance dark circular structures in dermoscopy images.

In this work we employ a technique described in [16], in order to detect dark linear structures in dermoscopy images. Let $\lambda_{1}, \lambda_{2}$ be the eigenvalues of $\mathbf{H}$ at any pixel $(x, y)$ with a specified value of $\sigma$. Since $\mathbf{H}$ is symmetric, both $\lambda_{1}, \lambda_{2}$ are real numbers. If the pixel $(x, y)$ belongs to a dark linear structure, then one of the eigenvalues of its Hessian matrix is of a positive and high value, whereas the other eigenvalue approaches zero. The eigenvalues $\lambda_{1}, \lambda_{2}$ can be calculated in closed form:

$$
\begin{aligned}
& \lambda_{1}=\frac{q}{a}, \lambda_{2}=\frac{D}{q} \\
& q=-\frac{1}{2}(b+\operatorname{sgn}(D)) \sqrt{T r^{2}-4 D}
\end{aligned}
$$

where $a=1, T r$ is the trace and $D$ the determinant of $\mathbf{H}$. Let $\lambda_{\max }$ be the maximum eigenvalue. The values $\lambda_{\max }$ for an exemplary dermoscopy image (Fig. 3a) for the pixels inside the lesion are shown in Fig. 3(b). Pixels with high value of $\lambda_{\max }$, are candidates for dark linear structures. The eigenvector, corresponding to the maximum eigenvalue, points to the direction of the maximum image curvature. If a pixel belongs to a linear structure, then the eigenvector with $\lambda_{\max }$ is directed perpendicular to the axis of the linear structure. The eigenvector $\mathbf{u}$ with $\lambda_{\max }$ is calculated as following:

$$
\mathbf{u}=\left(u_{x}, u_{y}\right), u_{x}=\frac{1}{\sqrt{1+\left(\frac{\lambda_{\max }-I_{x x}}{I_{x y}}\right)^{2}}}, u_{y}=\frac{\lambda_{\max }-I_{x x}}{I_{x y}} u_{x}
$$

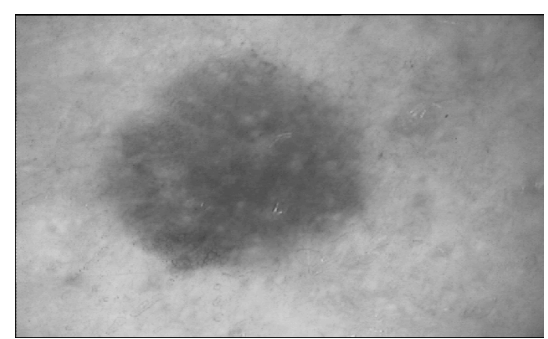

(a)

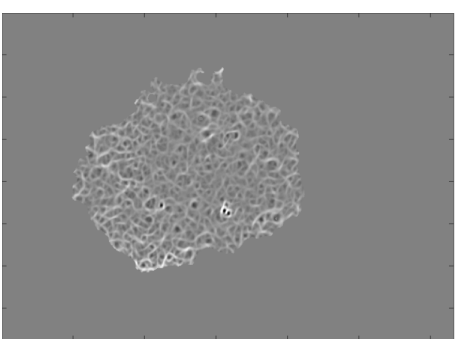

(b)

Fig. 3. The value of $\lambda_{\max }$ for the pixels inside the lesion (b) of an exemplary dermoscopy image (a). 
For any given pixel $(x, y)$, we calculate the exact point coordinates, where the $1^{\text {st }}$ order directional derivative along $\mathbf{u}$, vanishes and the $2^{\text {nd }}$ order directional derivative of the image, obtains high absolute value [16 Eqs. $(23,24)]$ :

$$
\left(p_{x}(x, y), p_{y}(x, y)\right)=\left(t(x, y) u_{x}(x, y), t(x, y) u_{y}(x, y)\right)
$$

where $t$ is given by

$$
t(x, y)=-\frac{I_{x}(x, y) u_{x}(x, y)+I_{y}(x, y) u_{y}(x, y)}{I_{x x}(x, y) u_{x}^{2}(x, y)+2 I_{x y}(x, y) u_{x}(x, y) u_{y}(x, y)+I_{y y}(x, y) u_{y}^{2}(x, y)}
$$

A pixel $(x, y)$ is considered as belonging to a dark linear structure, if the vanishing of the derivative occurs inside the pixel, or equivalently:

$$
\left\|\left(p_{x}(x, y), p_{y}(x, y)\right)\right\|_{2}<0.5 \Leftrightarrow|t(x, y)|<0.5,
$$

where \|\|$_{2}$ stands for the distance metric norm 2. Vector $\mathbf{u}$ is illustrated for all pixels that satisfy $\lambda_{\max }>0$, in the case of a dermoscopy image (Fig. 4(a)), as well as in the case of a simple image containing dark vessels (Fig. 5, left column). We used an image of vessels in order to clearly illustrate the accuracy of the aforementioned algorithm. To demonstrate the use of this algorithm in the case of dermoscopy images, vectors $\mathbf{u}$ are superimposed on magnified portions of dermoscopic lesions image, Fig. 4 (a), (b). Yellow dots in both examples indicate pixels that satisfy the criterion of Eq.(8).

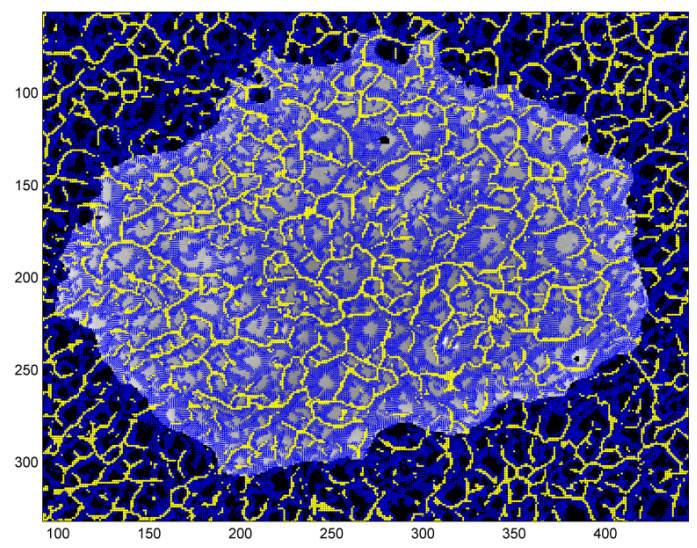

(a) 


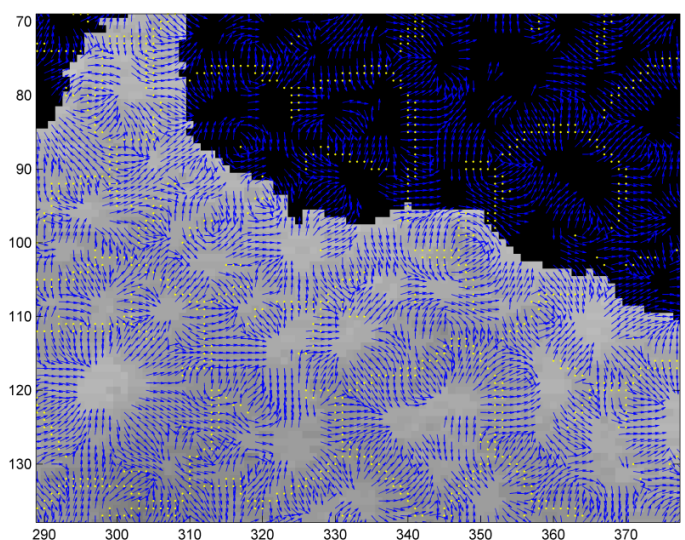

(b)

Fig. 4. Vectors $\mathbf{u}$ is superimposed on the pixels that satisfy $\lambda_{\max }>0$, for magnified portions (a) and (b) of the dermoscopy image in Fig.3(a), using $\sigma=3$.

\section{Multi-resolution approach}

The approach described above is parameterized by the $\sigma$ parameter of the Gaussian, as shown in Eqs. (2) and (3). The selection of $\sigma$ affects the size of the detected linear structures, as described in the theory of scale-space [14]. Thus, the above linear structure detection is applied to a given image at a different scales $\sigma_{i}$, with $i=1,2, \ldots, n$, producing different segmented images $B_{i}$ :

$$
B_{\iota}(x, y)=1 \Leftrightarrow\left\|\left(p_{x}\left(x, y ; \sigma_{i}\right), p_{y}\left(x, y ; \sigma_{i}\right)\right)\right\|_{2}<0.5
$$

Segmented images $B_{2}$ and $B_{3}$ (for $\sigma=2$ and $\sigma=3$ respectively) for the vessel image are shown in the right column of Fig.5.

The segmented images $B_{\mathrm{i}}$, produced by each $\sigma_{i}$ value, are further refined by applying hysteresis thresholding to the maximum eigenvalues of the image pixels. More specifically, high and low thresholds for $\lambda_{\max }(T H, T L$ respectively) are selected and the following process is performed for each image $B_{\mathrm{i}}$. A non-zero pixel $\left(x_{0}, y_{0}\right)$ of $B_{\mathrm{i}}$ is preserved if the following condition is met:

$$
\begin{aligned}
\lambda_{\max }\left(x_{0}, y_{0}\right) & \geq T H \text { OR } \\
& \left(\lambda_{\max }\left(x_{0}, y_{0}\right) \geq T L\right. \text { AND } \\
& \left.\left(x_{0}, y_{0}\right) \text { is connected to pixel with } \lambda_{\max } \geq T H \text { via pixels with } \lambda_{\max } \geq T L\right)
\end{aligned}
$$

Otherwise its value is set equal to 0 .

The final image with the linear segmented structures is obtained as the union of the segmented images $B_{\mathrm{i}}$ for the different values of $\sigma$, that have undergone hysteresis thresholding:

$$
B=B_{1} \cup B_{2} \cup \ldots B_{n}=\bigcup_{i=1}^{n} B_{i}
$$



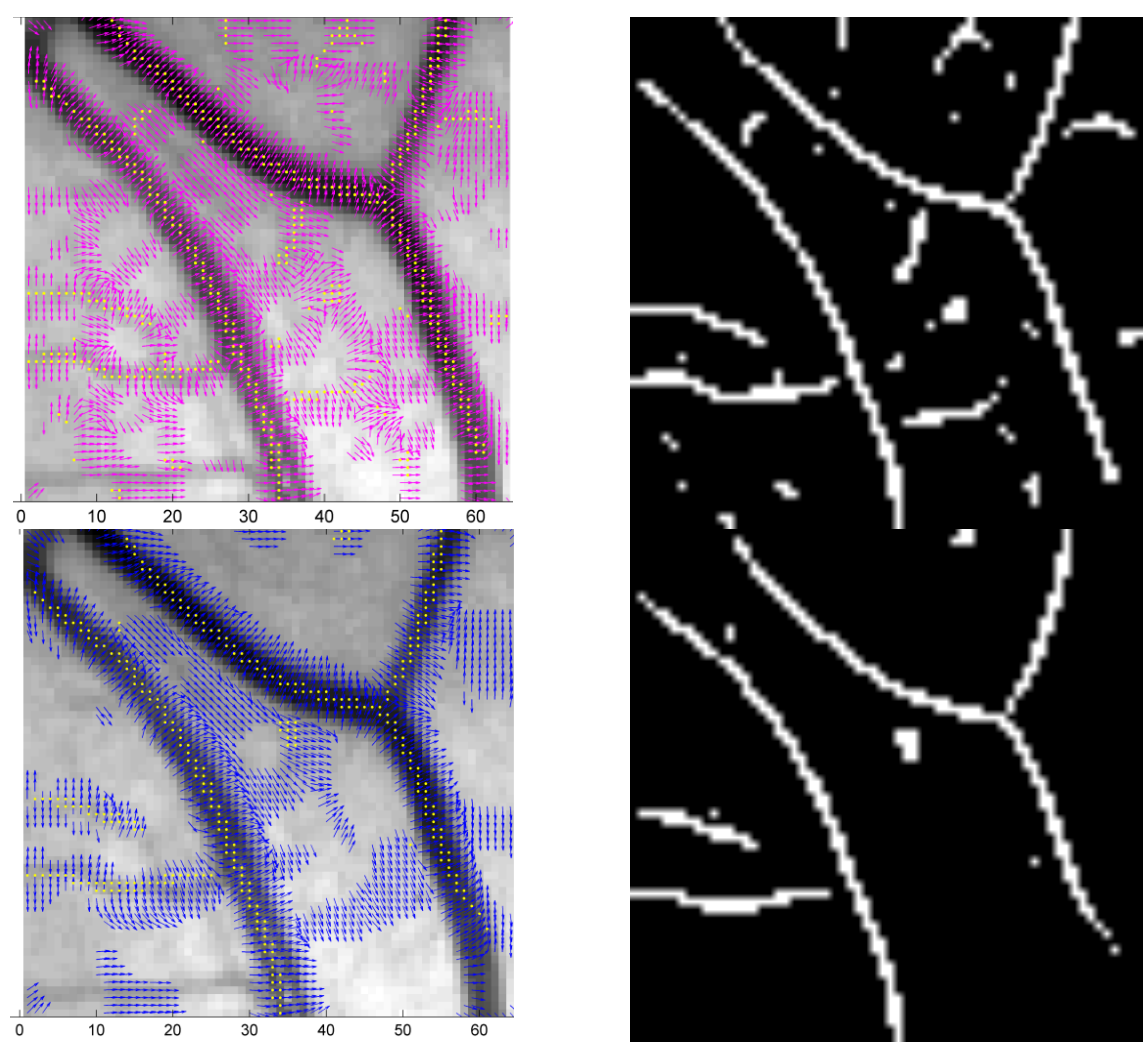

Fig. 5. Illustration of calculation of eigenvector for maximum eigenvalue (left column) in the case of an image with dark vessels, for $\sigma 2$ and 3 (upper and lower line, respectively). Right column shows the resulting vessel segmentation.

\subsection{Following and labeling of linear structures}

The previous algorithm creates a binary image, which contains pixels that belong to dark linear structures. The resulting image $B$ is skeletonized using a morphological algorithm. An exemplary dermatoscopy image of a lesion with the segmented linear structures superimposed as black pixels is shown in Fig. 6, (a) before and (b) after skeletonization. The junction pixels and the beginning/ending pixels of each streak are identified. This is performed using the following algorithm, implemented in [17]. Let us define the $3 \times 3$ neighborhood round each pixel as following:

\begin{tabular}{|l|l|l|}
\hline$v_{2}$ & $v_{1}$ & $v_{8}$ \\
\hline$v_{3}$ & $v_{0}$ & $v_{7}$ \\
\hline$v_{4}$ & $v_{5}$ & $v_{6}$ \\
\hline
\end{tabular}

A binary pixel with value $v_{0}>0$ is a junction if the following condition is fulfilled 


$$
\sum_{i=1}^{8}\left|v_{i}-v_{i+1}\right| \geq 6 A N D v_{0}>0
$$

A pixel with value $v_{0}>0$ is a beginning/ending pixel if the following condition is satisfied [15]

$$
\sum_{i=1}^{8}\left|v_{i}-v_{i+1}\right| \geq 2 A N D v_{0}>0
$$

The algorithm starts from a beginning/ending pixel and follows the segmented pixels until another beginning/ending pixel is found. The visited pixels are labeled as belonging to a linear structure. If a junction is found, the algorithm is re-initialized to track the new structure (as long as non-visited pixels are connected to the junction).

The linear structures outside the lesion of Fig. 3(a) are discarded (Fig.6, c) and the remaining structures are labeled by the algorithm. The resulting labeling is shown in color in Fig. 6(d).

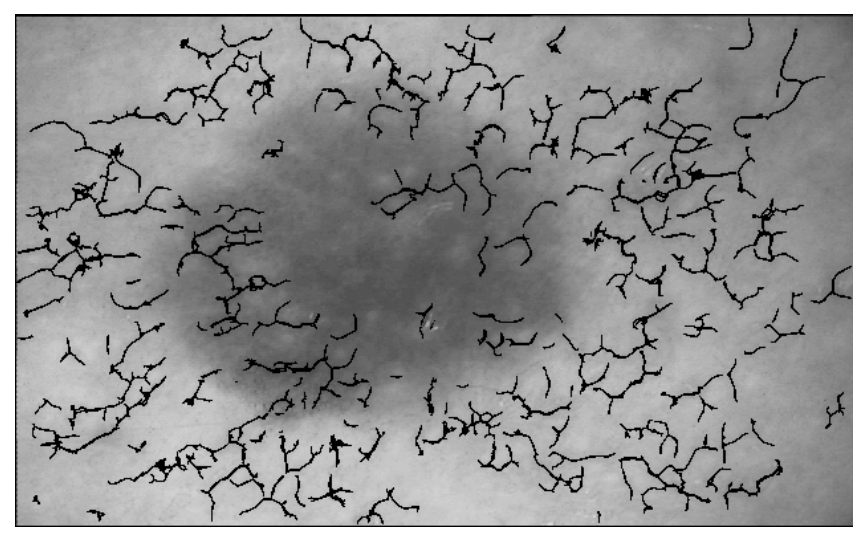

(a)

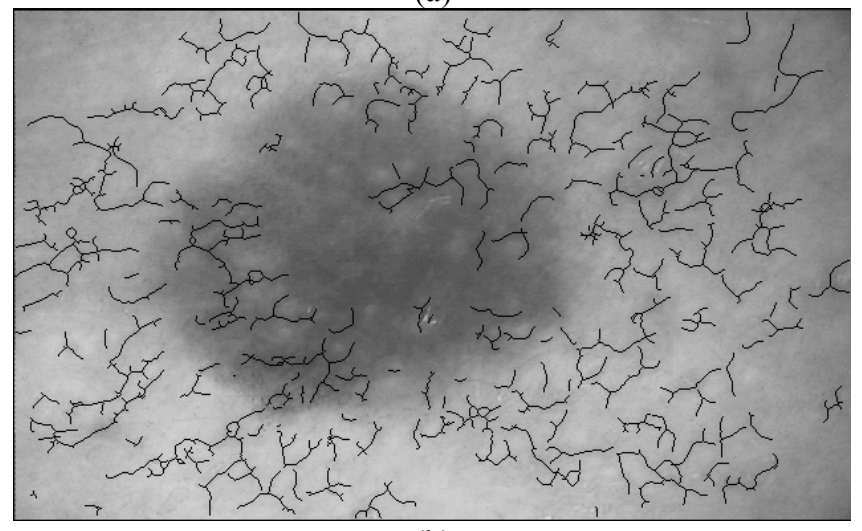

(b) 


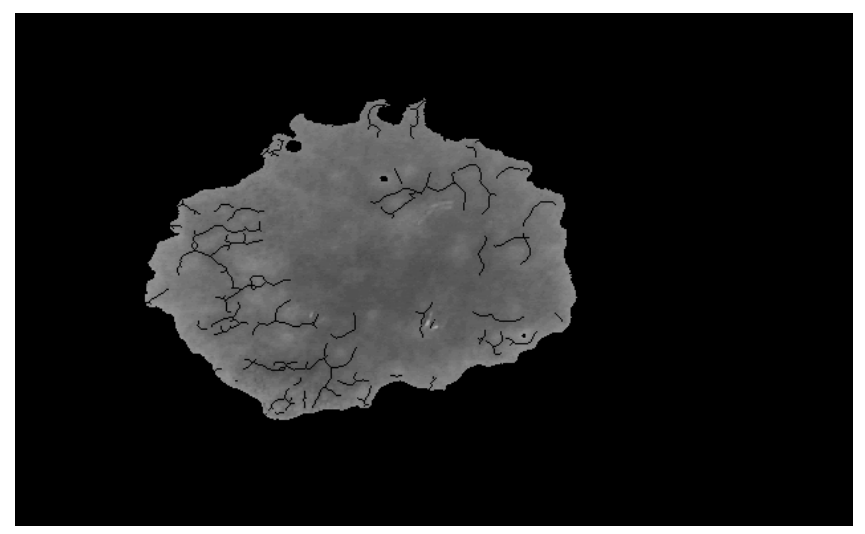

(c)

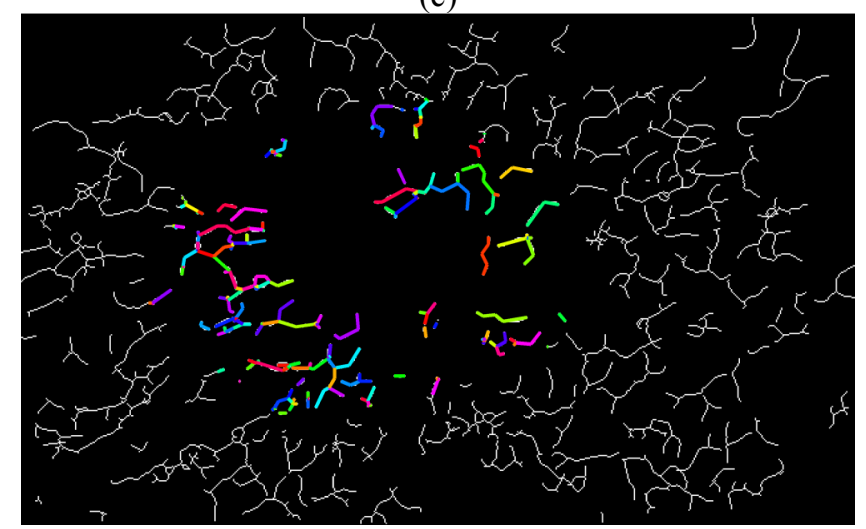

(d)

Fig. 6. The segmented linear structures (black pixels) from a dermatoscopy image of a lesion, (a) before and (b) after skeletonization. Skeletonized structures inside the lesion (c). The labeled linear structures that lie inside the lesion are shown in color in (d).

\subsection{Selection of streaks}

The geometric characteristics of streaks in dermoscopy images are summarized in [7, Introduction section], as following: dark linear structures, "co-radially oriented in the boundary", "darker than their neighborhood", "shorter than the 1/3 of the minor axis of the lesion and they should be longer than one percent of the major axis", and have low curvature. The segmented and labeled linear structures identified by the algorithm so far, are checked against these characteristics, as described below.

First the radial orientation of a linear structure is considered. In [8] this characteristic is checked by assuming the shape of the lesion to be elliptic and by calculating the orientation of the streak with respect to the two foci of the ellipse. However, in this work we utilize a simpler approach: we calculate the apparent angle $\varphi$ of the streak from the centroid of the skin lesion. Given the first $\left(x_{1}, y_{1}\right)$ and last pixel $\left(x_{N}, y_{N}\right)$ of a labeled linear structure (coordinates with respect to the centroid of the lesion), the apparent angle from the centroid is calculated as following: 


$$
\varphi=\left|\tan ^{-1} \frac{y_{n}}{x_{n}}-\tan ^{-1} \frac{y_{1}}{x_{1}}\right|
$$

If $\varphi$ is greater than a predefined threshold $\varphi_{t o l}$ then the linear structure is discarded.

$$
\varphi>\varphi_{t o l}
$$

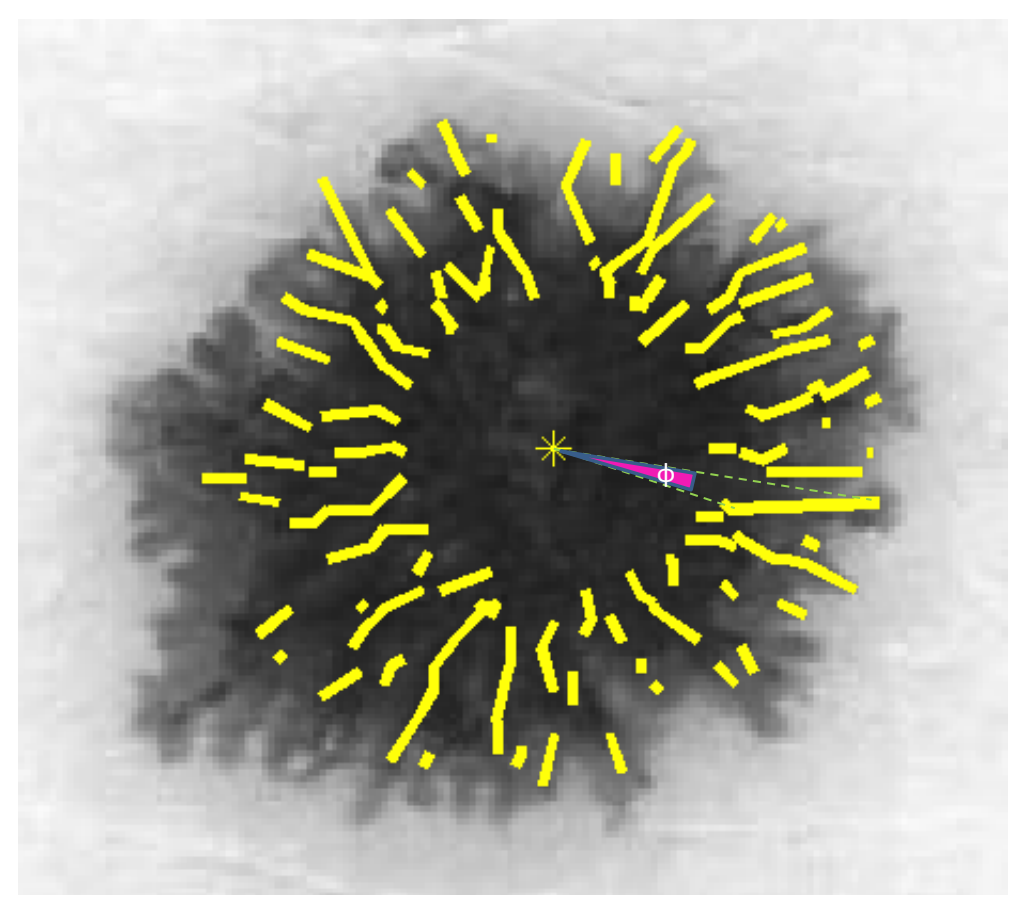

Fig. 7. An exemplar dermoscopy image with the detected streaks. The apparent angle $\varphi$ of a streak from the center of the lesion (marked by “*”) is shown.

The requirement for linear structures darker than the background is fulfilled by requiring positive eigenvalues of the $\mathbf{H}$ matrix. The upper and the low limit of the length of each linear structure require the calculation of the major and minor axis of the binary lesion, which is performed using the moments of inertia of the $2^{\text {nd }}$ order. An example of the calculation of the major axis is shown in Fig. 8. Thus a labeled linear structure is discarded if its length $L$ is smaller than 0.01 of the length of the major axis of the lesion $L_{1}$, or if its length is greater than $1 / 3$ of the minor axis $L_{2}$ of the lesion:

$$
L>\frac{1}{3} L_{2} \text { OR } L<0.01 L_{2}, L=\sqrt{\left(x_{1}-x_{N}\right)^{2}+\left(y_{N}-y_{1}\right)^{2}}
$$

Since the curvature of a linear structure should be small to be considered as streak, its length $L$ is calculated as the distance between beginning and ending point. $L$ is compared to major axis length in order to fulfill the relevant rule [7]. 


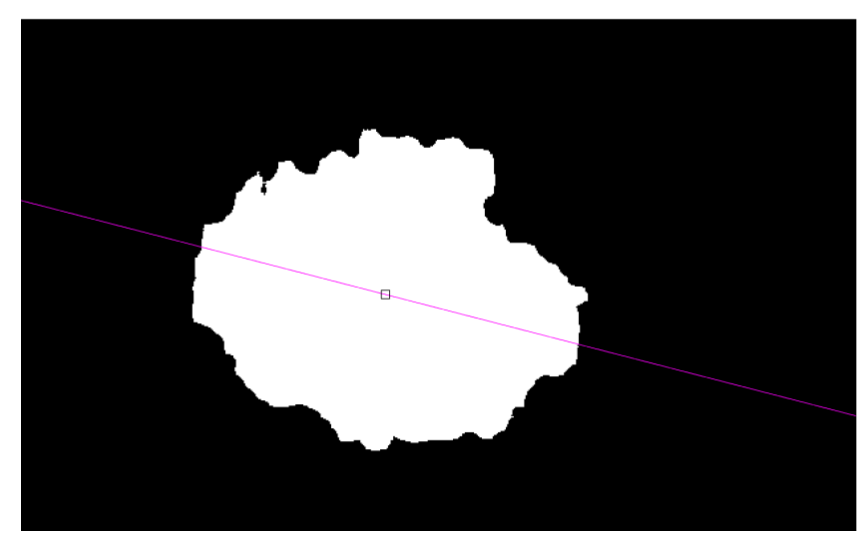

Fig. 8. An example of the calculation of the major axis of a binary lesion (continuous line). The centroid of the lesion is marked by a square.

In this study we seek to identify linear structures that resemble straight lines, thus structures with increased curvature need to be discarded. There are many approaches for curvature estimation of pixelized structures. We have utilized the simple but efficient approach implemented in [17], described as following:

For each linear binary object containing $N$ pixels $\left\{\left(x_{i}, y_{i}\right)\right\}, i=1,2, \ldots, N$, the equation of the line defined by its $1^{\text {st }}$ and last pixel $\left(x_{1}, y_{1}\right),\left(x_{N}, y_{N}\right)$ is considered and the maximum distance $d_{\max }$ of the distances $d_{i}$ of each pixel $\left(x_{i}, y_{i}\right)$ from the line was calculated:

$$
\begin{aligned}
& d_{i}=\left|x_{i} \delta y+y_{i} \delta x-C\right|, C=y_{N} x_{1}-y_{1} x_{N} \\
& d_{\max }=\max \left(d_{i}\right)
\end{aligned}
$$

If $d_{\max }$ is greater than a predefined threshold tol then the linear structure is discarded.

$$
d>d_{\text {tol }}
$$

According to [7], [8], streaks in dermoscopy images appear close to the boundary of the lesion. In this work, this characteristic is implemented by calculating the distance transform (DT) of the binary lesion.

A linear structure is discarded if the maximum distance of its two beginning/ending pixels from the boundary is greater than the (1/3) of the length of the minor axis $L_{2}$ of the lesion. Equivalently,

$$
\max \left(D T\left(x_{1}, y_{1}\right), D T\left(x_{N}, y_{N}\right)\right)>\frac{1}{3} L_{2}
$$




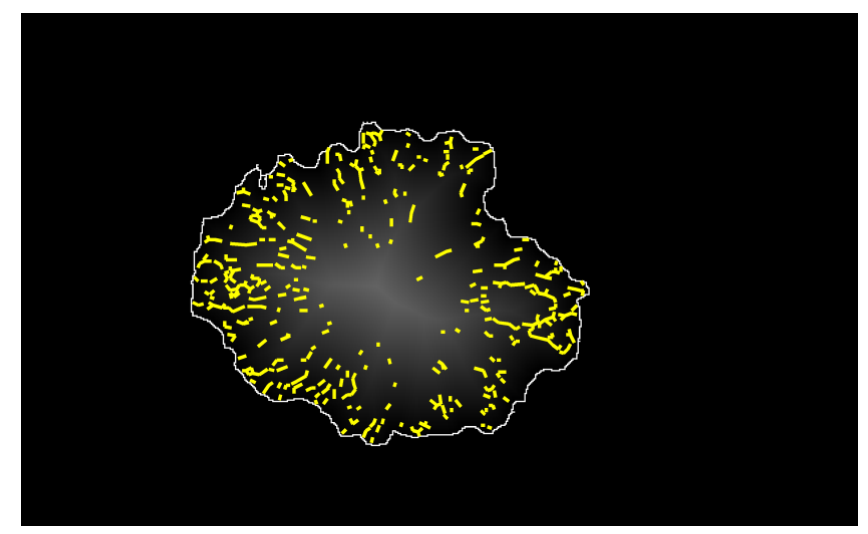

Fig. 9. The detected streaks from a dermoscopy image are shown, with the border of the lesion, as well as the distance transform of the border (encoded as shades of gray inside the lesion).

\section{Accepted streaks}

The linear structures that remain after discarding structures according to criteria in Eqs. (14), (15), (17) and (18) are considered as streaks.

\subsection{Extraction of streak-based features}

In [17] streaks are classified as regular or irregular according to their spatial distribution. In this work we define the angular and radial asymmetry of the spatial distribution of streaks with respect to the centroid of the lesion, as following. The centroid of each streak $\mathbf{c}_{\mathbf{i}}$ is located, defined as its median pixel. The azimuthial angle of the centroid $\theta_{i}$ and its distance from the boundary $r_{i}=\mathrm{DT}\left(\mathbf{c}_{\mathbf{i}}\right)$ (using the distance transform DT of the binary lesion) is calculated for each streak $i=1,2, \ldots, K$. The histogram of $\theta_{i}$ and $r_{i}$ is calculated ( $H_{\theta}$ and $H_{r}$ respectively) using 10 bins. The angular and radial asymmetry ( $A_{\theta}$ and $A_{r}$ respectively) are calculated as following:

$$
\begin{aligned}
& A_{\theta}=\frac{\max \left(H_{\theta}\right)-\min \left(H_{\theta}\right)}{\max \left(H_{\theta}\right)} \\
& A_{r}=\frac{\max \left(H_{r}\right)-\min \left(H_{r}\right)}{\max \left(H_{r}\right)}
\end{aligned}
$$

Both $A_{\theta}$ and $A_{r}$ are normalized in the [0,1], with values approaching 1 indicating high asymmetry. The number of detected streaks, as well as the number of pixels in detected streaks is also calculated and used as a feature. 


\section{Experimental Results}

The proposed algorithm was applied to 64 dermoscopy images with non-malignant lesions and to 35 images of malignant lesions, determined by histological analysis. All images have size of $632 \times 387$ pixels and the same magnification, resulting in spatial resolution of about $0.05 \mathrm{~mm} /$ pixel. They were acquired using the ELM Molemax II device at Hospital of Wien and at the department of Plastic Surgery and Dermatology in the General Hospital of Athens G. Gennimatas. The area of the lesion has been manually delineated for each one of the images. The same parameters of the algorithm were used for all images: $d_{\max }=2$ pixels, $\varphi_{t o l}=2 \mathrm{deg}, T H=0.1, T L=0.01$ (with respect to maximum $\lambda_{\max }$ value). The $\sigma$ parameter, described in subsections 2.1 and 2.2, takes integer values of 1, 2 and 3. The detected streaks are shown in Fig. 10 for exemplar non-malignant dermoscopy images (left column) and on malignant lesions (right column).
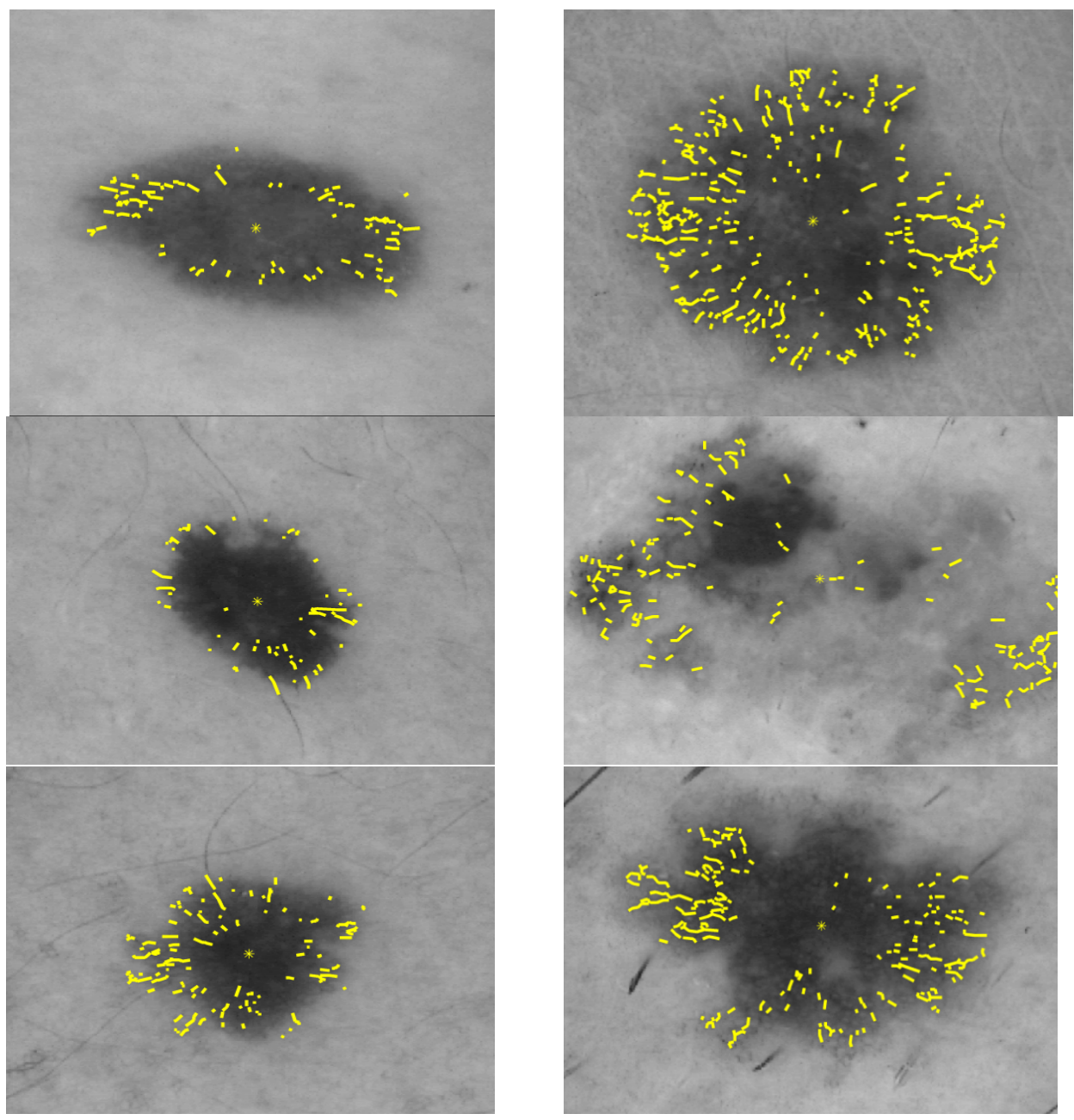


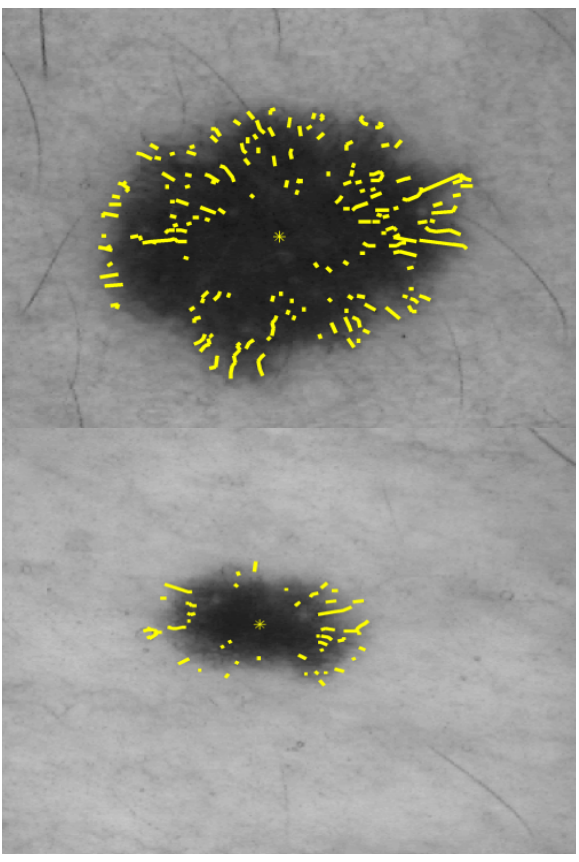

(a)

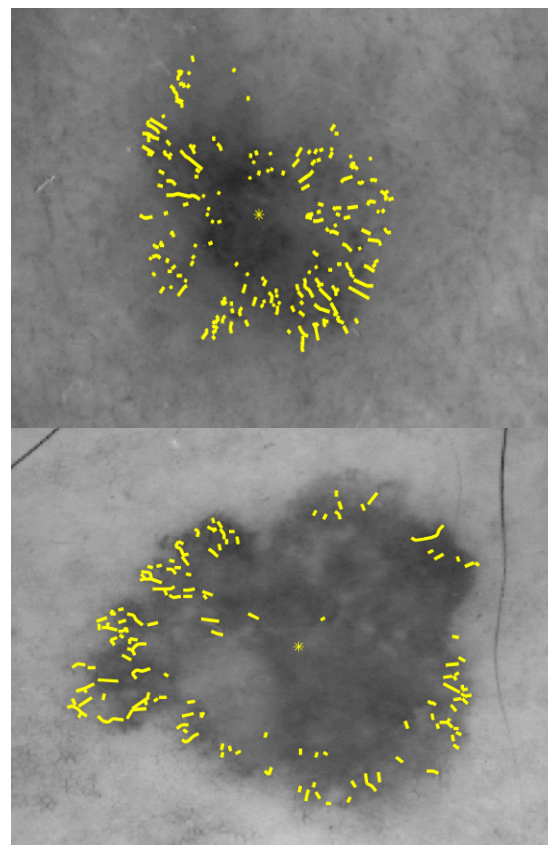

(b)

Fig. 10. Streaks detected by the proposed algorithm on non-malignant (a) and on malignant lesions (b). Images have been cropped, but are all in the same scale.

An initial effort to investigate the diagnostic value of streaks for the detection of malignancy in the skin lesions was undertaken. More specifically, the four streak-based features (described in Section 2.5) were used for classifying the dermoscopy images into 2 classes: "malignant" and "non-malignant". The ground truth of the specific lesion was determined by histological analysis as already mentioned. We have experimented with the following classifiers: the $k \mathrm{NN}$ (with $k=5$ ), the linear and quadratic discriminant, support vector machines (using Sequential Minimal Optimization), as well as feed forward neural networks with back error propagation, considering different number of neurons in the hidden layer.

Table 1. The classification results using the four streak-based features.

\begin{tabular}{|c|c|c|c|c|}
\hline \multicolumn{2}{|c|}{ Classification Method } & accuracy & sensitivity & specificity \\
\hline \multicolumn{2}{|c|}{$k \mathrm{NN}, k=5$} & 0.7022 & 0.600 & 0.7437 \\
\hline \multicolumn{2}{|l|}{$k \mathrm{NN}, k=7$} & 0.7040 & 0.3953 & 0.8294 \\
\hline \multicolumn{2}{|c|}{ Linear Discriminant } & 0.7249 & 0.7538 & 0.7131 \\
\hline \multicolumn{2}{|c|}{ Quadratic Discriminant } & 0.7000 & 0.7877 & 0.6644 \\
\hline \multicolumn{2}{|c|}{ Support Vector Machines } & 0.7511 & 0.6983 & 0.7731 \\
\hline Neural Nets & Hidden 9x1 & 0.6051 & 0.5757 & 0.6847 \\
\hline Neural Nets & Hidden $3 x 1$ & 0.5829 & 0.5804 & 0.5894 \\
\hline
\end{tabular}


The training set was 32 instances from non-malignant and 22 cases from malignant class. The achieved accuracy, sensitivity and specificity are shown in Table 1, averaged over 50 executions.

In addition to accuracy, sensitivity and specificity (true negative rate, complementary to false positive rate) are also presented. Sensitivity is of importance, since by its definition (true positive rate) is complementary to false negative rate, which is required to be low by any medical decision support system. The reported results prove the relevance of the streak-based features with skin lesion malignancy.

The complexity of the proposed algorithm is analogous to the total number of pixels in the dermoscopy images, as well as analogous to $\max \left(\sigma_{\mathrm{i}}\right)^{2}$, used in Eqs. (2), (3). Execution time was approximately equal to 2 seconds, for each dermoscopy image, with the parameters described above, using Matlab on an Intel(R) Core i5-2430 CPU (a) 2.40 GHz Laptop with 4 GB Ram, under Windows 7 Home Premium.

\section{Discussion and Conclusion}

An algorithm for the detection of streaks in dermoscopy images has been presented. The proposed methodology segments dark linear structures using Hessian-based multi-resolution image features. Qualitative results show successful detection of streaks. The quantitative results, concerning the classification of lesion into malignant or nonmalignant, according to features related to the detected streaks, appear promising. Although the support vector machines achieved the best overall accuracy $(75.11 \%)$, the highest sensitivity (equivalently lowest false negative rate) was achieved by the quadratic discriminant classifier.

It is in our future plans to continue this work by extending the dataset, as well as defining and utilizing more streak-based features, possibly in conjunction with other, already established features. Thus, we believe that the classification accuracy and sensitivity may increase further.

\section{Acknowledgments}

This work has been supported by the $12 \mathrm{CHN} 204$ Bilateral Greece-China Research Program of the Hellenic General Secretariat of Research and Technology and the Chinese Ministry of Research and Technology entitled "Personalization of melanoma therapeutic management through the fusion of systems biology and intelligent data mining methodologies-PROMISE," sponsored by the Program "Competitiveness and Entrepreneurship," Priority Health of the Peripheral Entrepreneurial Program of Attiki. 


\section{References}

1. Reed, K.B., Brewer, J.D.: Lohse CM, Bringe KE, Pruit CN, Gibson LE. Increasing Incidence of Melanoma Among Young Adults: An Epidemiological Study in Olmsted County, Minnesota. Mayo Clinic Proceedings 87(4), 328-334 (2012).

2. Stern, R.S.: Prevalence of a history of skin cancer in 2007: results of an incidence-based model. Arch Dermatol. 146(3), 279-282 (2010).

3. Rogers, H.W., Weinstock, M.A., Harris, A.R. et al.: Incidence estimate of nonmelanoma skin cancer in the United States, 2006. Arch Dermatol. 146(3), 283-287 (2010).

4. American Cancer Society. Cancer Facts \& Figures 2015. http://www. cancer.org/research/cancerfactsstatistics/cancerf actsfigures2015/ Accessed May 12, 2015

5. Maglogiannis, I., Doukas, C. N.: Overview of advanced computer vision systems for skin lesions characterization. IEEE Trans Inf Technol Biomed. 13(5), 721-33 (2009).

6. Korotkov, K., Garcia, R. Computerized analysis of pigmented skin lesions: A review. Artificial Intelligence in Medicine 56 (2), 69-90 (2012).

7. Sadeghi, M., Lee, T. K., McLean, D., Harvey Lui, Atkins, M. S.: Oriented Pattern Analysis for Streak Detection in Dermoscopy Images. In: N. Ayache et al. (Eds.): MICCAI 2012, Part I, LNCS 7510, pp. 298-306 (2012).

8. Sadeghi, M., Lee, T. K., McLean, D., Lui, H., Atkins, M. S.: Detection and Analysis of Irregular Streaks in Dermoscopic Images of Skin Lesions, IEEE T on Medical Imaging 32(5), 849-861 (2013).

9. Mikolajczyk, K., Tuytelaars, T., Schmid, C., Zisserman, A., Matas, J., Schaffalitzky, F., Kadir, T., Van Gool, L.: A Comparison of Affine Region Detectors. International Journal of Computer Vision 65(1-2), 43-72 (2005).

10. Tuytelaars, T., and Mikolajczy, K.: Local Invariant Feature Detectors: A Survey. Computer Graphics and Vision 3(3), 177-280 (2007).

11. Frangi, A., Niessen, W. J., Vincken, K. L., Viergever, M. A.: Multiscale vessel enhancement filtering. In: Proceedings of the 1st International Conference of Medical Image Computing and Computer-Assisted Intervention (MICCAI 1998), pp. 130-137, (1998).

12. Aylward, S., Bullitt, E.: Initialization, noise, singularities, and scale in height-ridge traversal for tubular object centerline extraction. IEEE Transactions on Medical Imaging 21, 61-75 (2002).

13. Martinez-Perez, E., Hughes, A., Thom, S., Bharath, A., Parker, K.: Segmentation of blood vessels from red-free and fluorescein retinal images. Medical Image Analysis 11, $47-61$ (2007).

14. Lindeberg, T.: Edge detection and ridge detection with automatic scale selection. International Journal of Computer Vision 30(2), 117-156, (1996).

15. Maglogiannis, I., Delibasis, K.: Enhancing classification accuracy utilizing globules and dots features in digital dermoscopy. Computer Methods and Programs in Biomedicine 118(2), 124-133 (2015).

16. Steger, C.: Extracting lines using differential geometry and Gaussian smoothing. International archives of Photogrammetry and Remote sensing 16 (B3), 821-826 (Vienna 1996).

17. Kovesi, P.: MATLAB and Octave functions for computer vision and image processing. Centre for Exploration Targeting, The University of Western Australia, http: //www.csse. uwa.edu.au/ pk/research/matlabfns/ 